\title{
Peroneal nerve palsy caused by a synovial cyst of the proximal tibiofibular joint: A case report
}

\author{
Swantje M. Kruspi, Michael Dietrich \\ Stadtspital Waid, Department of Surgery, Switzerland
}

Received: May 17, 2018

Accepted: July 25, 2018

Online Published: August 6, 2018

DOI: $10.5430 /$ css.v4n2p11

URL: https://doi.org/10.5430/css.v4n2p11

\begin{abstract}
Peroneal nerve entrapment is the most common entrapment found in the lower limb, even though nerve palsy caused by a synovial cyst of the proximal tibiofibular joint (PTFJ) is a very rare condition. We report the case of a 54-year-old man who developed sudden onset of an incomplete foot droop and therefore presented himself to the emergency room. Further examination showed a compression of the peroneal nerve provoked by a synovial cyst of the PTFJ. Treatment involved puncture of the cyst. The patient showed a complete and fast recovery. We discuss our case with regards to the recent literatures.
\end{abstract}

Key Words: Cyst of the proximal tibiofibular joint, Atraumatic peroneal nerve entrapment, Foot droop

\section{INTRODUCTION}

Peroneal nerve entrapment is the most common entrapment found in the lower limb. ${ }^{[1,2]}$ Cysts in the knee region are mostly synovial cysts located in the popliteal region and were first described by Adams in 1840 and later in more detail by Baker in 1877.

Synovial cysts are defined as benign soft-tissue tumors, which originate from the synovial joints or tendon sheaths. ${ }^{[3]}$ Such cysts can show an infiltrative nature. Leander first reported a cyst of the proximal tibiofibular joint (PTFJ) in 1891. ${ }^{[4]}$

The following case report shows an uncommon presentation of this rare condition.

\section{CaSe report}

A 54-year-old man presented himself to the emergency department with aching muscles in the right calf and numbness of the right dorsal surface of the foot. The patient was work- ing as a truck driver. Due to a malfunction of the hydraulic ramp he had to jump up and down the ramp for the last four days. The muscles of the right calf started aching afterwards and he described a numbness of his foot and lower leg. Physical examination showed a hematoma about $5 \mathrm{~cm}$ under the fibular head and a palpable, immovable hard mass. Furthermore, the examination revealed a paresis of the M. tibialis anterior, M. extensor digitorum longus and M. extensor halluces longus with muscle strength of M3 according to Janda. Hypesthesia at the anterolateral side of the lower leg and the dorsal surface of the foot was also found. The sensitivity of the deep peroneal nerve was intact. The examination of the right knee showed no abnormalities.

The ultrasound performed did not provide further information, but a CT-scan showed a multilobular cyst of the PTFJ. The cyst measured $2 \mathrm{~cm} \times 1.7 \mathrm{~cm} \times 4.5 \mathrm{~cm}$ and was enclosed by an edema in the musculus peroneus longus. An MRI was performed to clarify if this cyst was in close proximity to the common peroneal nerve (see Figure 1). Therapy

*Correspondence: Swantje M. Kruspi; Email: Swantje.kruspi@waid.zuerich.ch; Address: Tièchestrasse 99, 8037 Zürich, Switzerland. 
was initiated by puncturing the cyst, draining $4 \mathrm{ml}$ of viscous, clear fluid. Microscopy revealed a cell number of $0.3 \mathrm{G} / \mathrm{l}$ and no crystals were found. Bacterial cultures were negative.

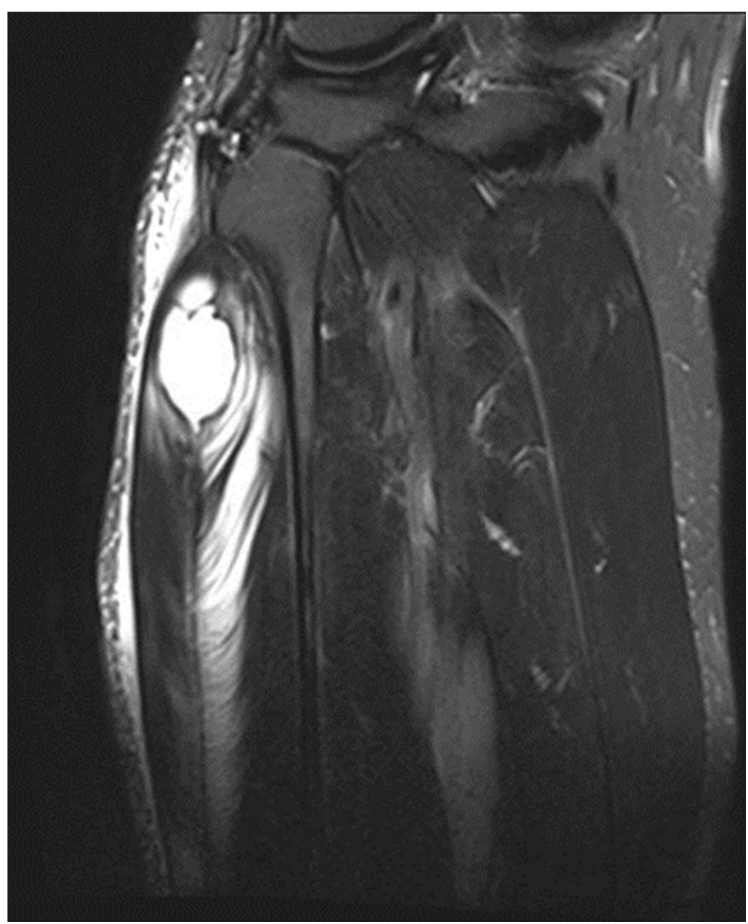

Figure 1. Initial MRI showing a multilobular cyst of the proximal tibiofibular joint with edema in the muscles peroneus longus

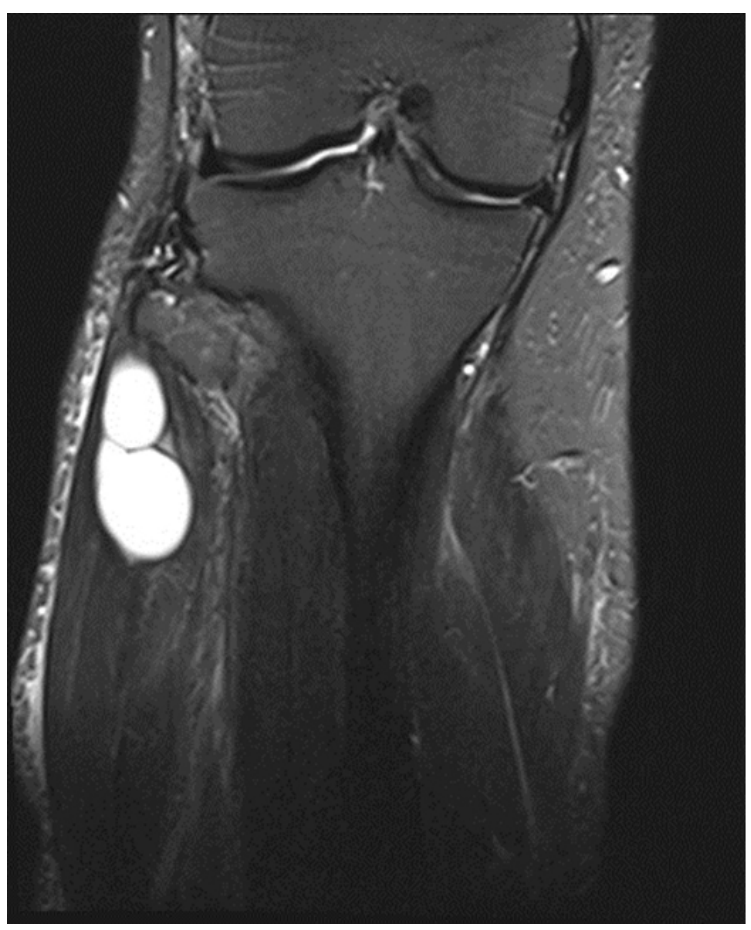

Figure 2. MRI after 4 weeks. The multilobular cyst still present, while the edema has gone completely
The patient was referred to a neurologist, who diagnosed by electroneuromyography an incomplete peripheral neuropathy of the $\mathrm{N}$. peroneus communis with intact continuity of the nerve.

Only 10 days after puncture of the cyst the hypesthesia of the anterolateral side of the lower leg was gone, but the patient still had incomplete muscle strength of the extensors of the foot, which were still M3 according to Janda.

At a neurologic follow up after 4 weeks including MRI (see Figure 2) a partial axonal lesion of the peroneal nerve was diagnosed. Three months after the intervention muscle strength was M5 according to Janda (see Figure 3) The patient had already returned to work one month ago.

Written evaluation after one year showed that the patient was still free of symptoms. He reported to feel pain only after strenuous working days, during which he would experience lower leg aches. Neurological symptoms did not reoccur.

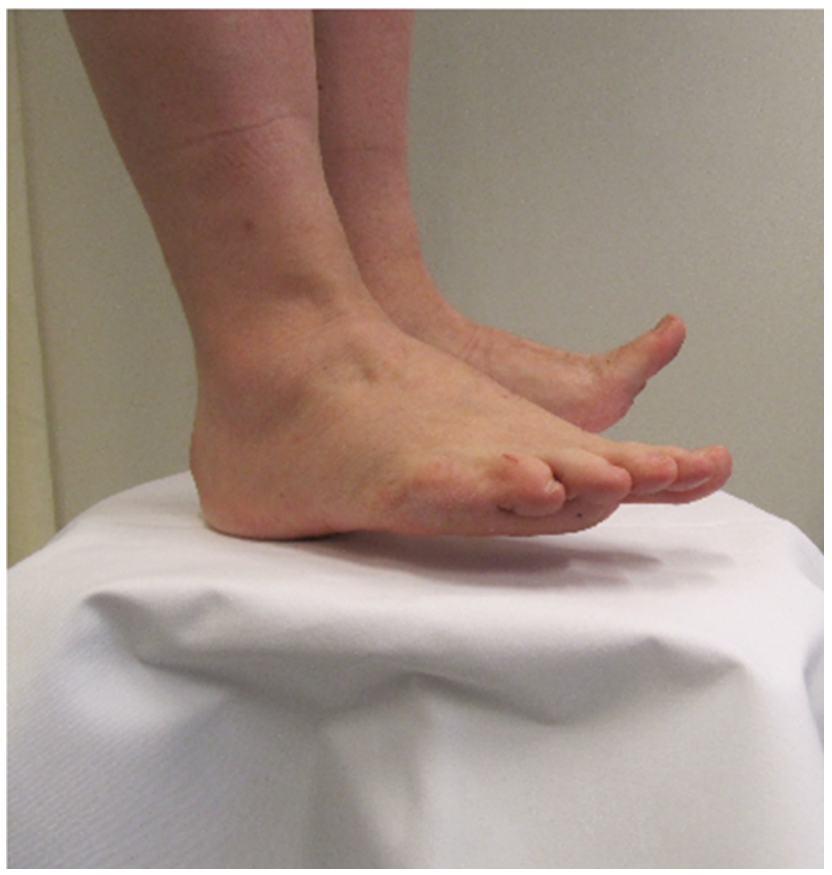

Figure 3. After 3 months the muscle strength of the foot recovered

\section{Discussion}

To the best of our knowledge there are only 73 reported cases of peroneal nerve palsy caused by cysts of the PTFJ since 1891. However, differences in histological definitions and artificial distinctions that exist between synovial cysts and ganglia have complicated comparison of published data. ${ }^{[5]}$

Synovial cysts of the PTFJ are rare. ${ }^{[6,7]}$ In a study by Ilahi et al. involving knee MRIs of 654 patients, only 5 patients 
showed a cyst and just one had lateral knee symptoms. However, it remains possible that these symptoms may have arisen from an additional lateral meniscus lesion. This corresponds to a prevalence of $0.76 \%{ }^{[6]}$

In our case the electroneuromyography showed a partial axonal lesion, which classified the peroneal nerve palsy as a nerve injury grade II according to Sunderland. This is associated with a full recovery potential in 2 to 4 months. ${ }^{[1]}$

Surgical decompression should be considered for compressive lesions at the fibular head or tunnel. ${ }^{[1]}$ While the time window for decompression is unclear, an improvement in nerve function can be seen for up to 6 months.

Regarding the common management of peroneal nerve palsy most authors suggest a minimum of 3 months of conservative treatment. ${ }^{[1]}$ Since our patient recovered within the first three months, no surgical decompression of the nerve was needed.

In terms of imaging techniques there seems to be a consensus favoring MRI. ${ }^{[8-10]}$ MRI shows the soft tissue, the communication to the joint through a pedicle and the extraor intraneural location of the cyst.

Since MRI discriminates poorly between cysts and soft tissue tumors, ultrasonography may be helpful in unclear cases. ${ }^{[10]}$ Differential diagnoses include juxtaarticular or intraarticular myxoma, schwannoma, neurofibroma or synovial sarcoma.

While reviewing the literature, it seems that in some cases therapy consisted in needle aspiration and injection of cortisol into the cyst. ${ }^{[7,10]}$

Others describe a high recurrence rate after needle aspiration and therefore prefer excision of the cyst as treatment of choice. ${ }^{[10]}$ However, we were unable to find reliable data on the recurrence rate after needle aspiration. The recurrence rate after surgical resection is described as less than $10 \% .{ }^{[4]}$ It seems that in the last two decades the preferred treatment is excision of the cyst, when the patient has symptoms. ${ }^{[3-5,8-13]}$ Gulati et al. even reported a case series with nine patients with recurrent ganglion cysts, in which proximal fibulectomy was performed, after either repeated needle aspirations or excisions of the ganglion cysts had already taken place. ${ }^{[14]}$

Others describe the necessity to remove the pedicle between the cyst and the joint. ${ }^{[1,9,10]}$ This pedicle is described in $20 \%-50 \%$ of the cases. ${ }^{[4]}$ Some authors claim that synovial cysts are arising from an increase of synovial fluid, which is causing a higher intraarticular pressure and therefore leading to a herniation or distension of the capsule. ${ }^{[12]}$ Therefore, a pedicle must always be present. In their opinion the pedicle has to be found and needs to be removed. ${ }^{[4]}$

Since synovial cysts causing peroneal nerve palsy are so far Published by Sciedu Press described in case reports and case series, there is no standard treatment established yet. The various treatment modalities described in literature included needle aspiration and steroid administration, arthroscopic removal, open excision and proximal fibulectomy. There is also no empiric data on the results and risks of each treatment modality available from the literature.

We recommend that needle aspiration should be guided by ultrasound or CT scan to avoid incomplete aspiration which may be one of the reasons for the high recurrence rate described. The through needle aspiration possible risk of a nerve damage caused by a cyst in close proximity to a neurovascular bundle can be reduced. ${ }^{[15]}$ One of the drawbacks of this technique is that the pathogenesis of the cyst, which is described by some authors as pedicle, is not treated and therefor the cyst can fill up again. ${ }^{[15]}$ Another risk to be considered is the occurrence of postinterventional septic arthritis due to the connection to the joint caused by the pedicle. The infection rate of needle aspiration should be comparable to an intraarticular injection, which lies between $0.003 \%$ and $0.34 \%$. The risk rises with additional steroid infiltration.

However the infection rate after a diagnostic arthroscopy is described between $0.1 \%$ and $0.42 \%$. In general arthroscopic surgery the infection rates lies by $0.04 \%$ to $0.28 \%$, which should be comparable to the removal of a cyst of the PTFJ. ${ }^{[16]}$

This leads to the assumption that an open excision is connected to a higher rate of septic arthritis, which can cause cartilage destruction within 1-2 days and is associated with a considerable mortality rate up to $11 \% .{ }^{[17]}$ The advantage of an open excision is the removal of a pedicle, if it can be clearly identified. An open excision furthermore has the risk of lesions to the neurovascular structures and should therefore be performed by an experienced surgeon only and nerve monitoring should be considered. ${ }^{[1]}$

Proximal fibulectomy is commonly used in proximal fibula tumors. In these cases postoperative permanent peroneal nerve palsy was found in $3 \%$, whereas knee instability was only temporary. ${ }^{[18]}$

\section{Conclusions}

Peroneal nerve palsy caused by a synovial cyst of the PTFJ is a very rare disease which must be considered if a patient presents neurological abnormalities of the lower leg. Recent literature is in favor of an operative treatment in patients with symptomatic cysts of the PTFJ, but there are still significant recurrent rates combined with an operative risk to be taken into account. Recovery rates are sometimes unclear since they depend on the date of the operative treatment and on the kind of preexisting nerve injury. Therefore, we suggest 
needle aspiration as an option even in patients with a recent onset of neurological symptoms. A close follow up is needed to see if the patient recovers within 3 months. If this is not the case surgical treatment is definitely to be considered. However, our case shows that this is not always necessary.

\section{REFERENCES}

[1] Poage C, Roth C, Scott B. Peroneal Nerve Palsy: Evaluation and Management. J Am Acad Orthop Surg. 2016; 24(1): 1-10. https: //doi.org/10.5435/JAAOS-D-14-00420

[2] Butler M, Talley KMC, Burns R, et al. Values of Older Adults Related to Primary and Secondary Prevention. U.S. Preventive Services Task Force Evidence Syntheses, formerly Systematic Evidence Reviews. Rockville (MD). 2011

[3] Byvaltsev VA, Belykh EG, Baradieva PJ, et al. Synovial cyst that compressed the peroneal nerve: case report. Acta Neurochir (Wien). 2010; 152(7): 1241-4. https://doi.org/10.1007/s00701-009 $-0567-z$

[4] Mortazavi SM, Farzan M, Asadollahi S. Proximal tibiofibular joint synovial cyst-one pathology with three different presentations. Knee Surg Sports Traumatol Arthrosc. 2006; 14(9): 875-9. https ://doi org/10.1007/s00167-005-0021-6

[5] Spinner RJ, Hebert-Blouin MN, Amrami KK. A complex cyst characterized into its individual components: a shared pathogenesis from the superior tibiofibular joint. J Surg Orthop Adv. 2010; 19(3): 143-8. PMid: 21086925.

[6] Ilahi OA, Younas SA, Labbe MR, et al. Prevalence of ganglion cysts originating from the proximal tibiofibular joint: A magnetic resonance imaging study. Arthroscopy. 2003; 19(2): 150-3. https://doi.org/10.1053/jars.2003.50007

[7] Gibbon AJ, Wardell SR, Scott RD. Synovial cyst of the proximal tibiofibular joint with peroneal nerve compression after total knee arthroplasty. J Arthroplasty. 1999; 14(6): 766-8. https: //doi.org/10.1016/S0883-5403(99)90235-8

[8] Pecina HI, Boric I, Pecina TC, et al. Double synovial cyst of the proximal tibiofibular joint confirmed by MRI as a cause of the peroneal tunnel syndrome. Acta Chir Orthop Traumatol Cech. 2008; 75(4): 301-5. PMid: 18760088

\section{ACKNOWLEDGEMENTS}

We acknowledge the assistance of T. Betschart, MD of the Department of Radiology for his assistance in radiologic interpretation.

\section{CONFlicts OF INTEREST Disclosure}

The authors declare they have no conflicts of interest.
[9] Pagnoux C, Lhotellier L, Marek JJ, et al. Synovial cysts of the proximal tibiofibular joint: three case reports. Joint Bone Spine. 2002; 69(3): 331-3. https://doi.org/10.1016/S1297-319X (02)0 0403-7

[10] Jerome D, McKendry R. Synovial cyst of the proximal tibiofibular joint. J Rheumatol. 2000; 27(4): 1096-8. PMid: 10782844.

[11] Zumrut M, Demirayak M, Kucukapan A. An Unusual Cause of Foot Drop: Peroneal Extraneural Ganglion Cyst. Pak J Med Sci. 2016; 32(4): 1047-50

[12] Bianchi S, Abdelwahab IF, Kenan S, et al. Intramuscular ganglia arising from the superior tibiofibular joint: CT and MR evaluation. Skeletal Radiol. 1995; 24(4): 253-6. PMid: 7644935. https : //doi.org/10.1007/BF00198408

[13] Hersekli MA, Akpinar S, Demirors H, et al. Synovial cysts of proximal tibiofibular joint causing peroneal nerve palsy: report of three cases and review of the literature. Arch Orthop Trauma Surg. 2004; 124(10): 711-4. https://doi.org/10.1007/s00402-004-071 $7-\mathrm{y}$

[14] Gulati A, Lechler P, Steffen R, et al. Surgical treatment of recurrent proximal tibio-fibular joint ganglion cysts. Knee. 2014; 21(5): 932-5. https://doi.org/10.1016/j.knee.2014.05.002

[15] Sinha K, Maheshwari R, Agrawal A. Giant synovial cyst of thigh: a rare entity. Case Rep Orthop. 2013; 2013: 967215.

[16] Stutz G. Diagnostik und arthrokopische Therapie von Gelenksinfekten. SFA Arthroskopie Aktuell. 2005; 18: 4-16.

[17] Munoz-Egea MC, Blanco A, Fernandez-Roblas R, et al. Clinical and microbiological characteristics of patients with septic arthritis: A hospital-based study. J Orthop. 2014; 11(2): 87-90.

[18] Abdel MP, Papagelopoulos PJ, Morrey ME, et al. Surgical management of 121 benign proximal fibula tumors. Clin Orthop Relat Res. 2010; 468(11): 3056-62. 